\title{
Noncompaction of the Left Ventricle: Report of Two Cases with Electron Microscopic Findings
}

\author{
S. Siew, ${ }^{*}$ M.A. Markey**
}

* Division of Human Pathology, College of Osteopathic Medicine, Michigan State University, East Lansing, MI 48824

** Sparrow Forensic Services, 1215 East Michigan Avenue, Lansing, MI 48909

The primitive heart is avascular. The internal endothelial tube continues as the principal constituent of the endocardium. The investing folds of splanchnic mesoderm transform into both the myocardium and epicardium. The myocardial coat differentiates into a thin cortical layer of dense muscle and a thick inner spongy layer of loosely arranged trabeculae, which project into the heart cavity. The endocardium dips down into the spaces between the trabeculae and wraps around them. The trabecular structure of the myocardium facilitates oxygen diffusion from the blood passing through its chambers. There is a gradual increase in thickness of the compact layer and oxygen diffusion from the lumen becomes inadequate. Endothelial lined channels (embryonal sinusoids) form throughout the myocardium. Strands of these tubes encircle the root of the aorta and penetrate it to form the coronary ostia. Between 5 to 8 weeks of gestation, the myocardium transforms into a compacted structure, concomitant with the development of the coronary arteries. The compaction begins at the base of the heart and proceeds towards the apex. Premature cessation of embryonic morphogenesis results in persistence of embryonal sinusoids and hypertrabeculation, which is most marked at the apex of the left ventricle, giving rise to a spongy appearance on gross examination. Noncompaction of the myocardium results in perfusion defects in the zones of noncompacted myocardium. Left ventricular noncompaction is commonly associated with progressive dysfunction and tachyarrhythmias. There may be both systolic and diastolic heart failure and thromboembolic complications.

We report 2 cases of noncompaction of the left ventricle.

CASE I : A 10 year old girl with known heart disease since infancy presented in severe respiratory distress and heart failure. Echocardiography showed the presence of marked cardiac enlargement and, possibly, mural thrombi.

At autopsy there was cardiomegaly, noncompaction of the apical segment of the left ventricle, endocardial fibroelastosis and a mural thrombus as shown in Fig. 1. It is interesting to speculate whether the endocardial fibroelastosis prevented compaction of the myocardium or was secondary to the noncompaction.

CASE II : A 5 month old boy-one of twins, born at 37 weeks of gestation via Caesarean section had feeding difficulties and mild hypotonia. Developed respiratory distress and became listless. Chest $\mathrm{x}$ ray showed cardiomegaly.

At autopsy, the heart was enlarged, with thickening and, hypertrabeculation of the myocardium, which had a diffuse, tan yellow appearance. Transmission electron microscopy showed the presence of persistent dilated embryonal sinusoids, surrounded by fetal myoblasts as shown in Fig. 2. To our knowledge, this is the first report of the electron microscopic findings of noncompaction cardiomyopathy. 


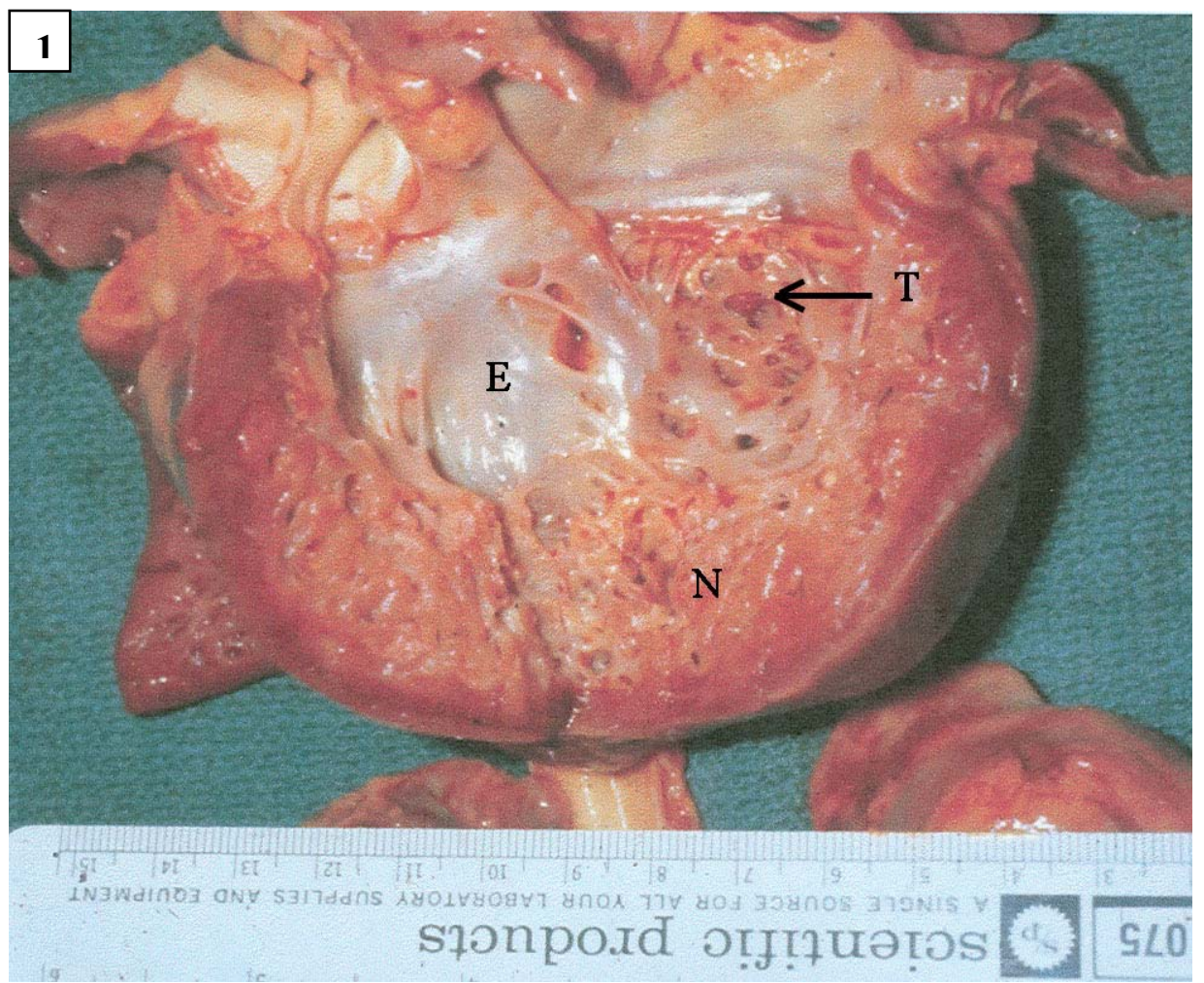

FIG. 1. Heart of Case 1. E-Endocardial Fibroelastosis;N-Noncompaction;T-Mural Thrombus

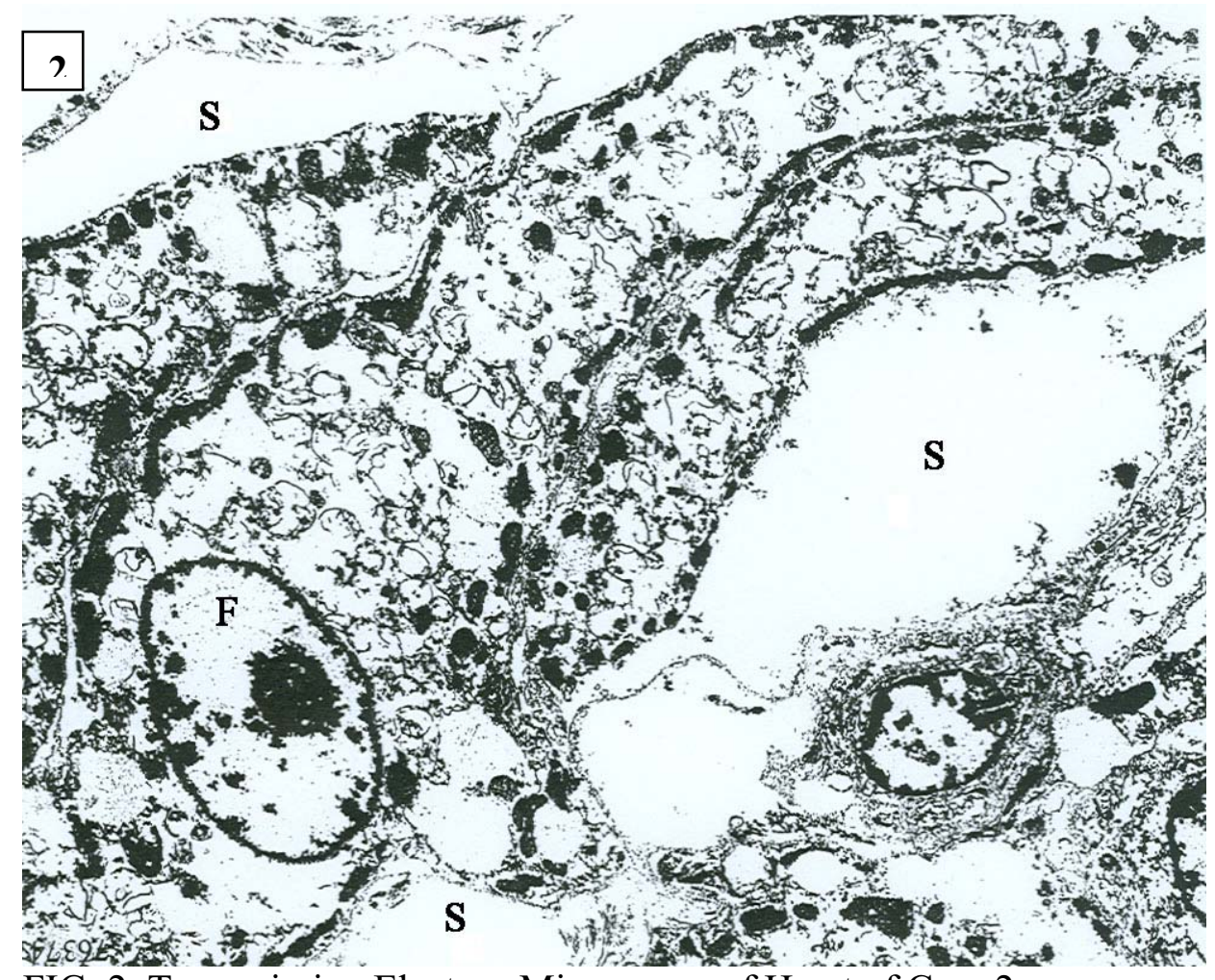

FIG. 2. Transmission Electron Microscopy of Heart of Case 2.

F-Fetal myoblast;S-Embryonal Sinusoids Original Magnification-2,500x. 